\title{
Música e exercício físico: revisão de literatura
}

\author{
Larissa Pereira Cova ${ }^{1}$ \\ Gabriela Kaiser Fullin Castanho ${ }^{2}$ \\ Paula Teixeira Fernandes ${ }^{1}$
}

\section{Resumo}

Independente de nossas origens e contextos culturais, a música apresenta papel fundamental em nossas vidas. A música é capaz de alterações psicofisiológicas com aplicações no esporte e exercício. O objetivo do presente estudo é revisar o conhecimento científico a respeito da possível influência da música no esporte e exercício. Selecionamos artigos presentes na base de dados Physical Education Index, publicados entre os anos 2010 e 2015 e que foram revisados por especialistas. Foram excluídos aqueles que não tratavam do tema da presente pesquisa, assim analisamos 13 artigos. De acordo com os estudos revisados, a música parece beneficiar de forma aguda os exercícios físicos de caráter aeróbio, diferentemente dos exercícios anaeróbios, o que se deve provavelmente à alta intensidade. Apesar disso, sua eficácia para a aplicação no esporte, sobretudo no de alto rendimento ainda não está completamente clara.

Palavras-Chave: Música. Exercício. Esporte. Rendimento.

1 Universidade Estadual de Campinas

${ }^{2}$ Universidade Estadual Paulista

Submetido em: 20 set. 2016

Aceito em: 02 maio 2017

Contato: larissapereiracova@ hotmail.com

Conexões: Educ. Fís., Esporte e Saúde, Campinas: SP, v. 15, n. 2, p. 200-209, abr./jun. 2017. ISSN: 1980-9030 


\section{The music in the sport: literature review}

\section{A BStraCT}

Regardless of our origins and cultural contexts, the music represents fundamental role in our lives. The music is able to psycho physiological changes with applications in the sport and exercise. The aim of this study is to review the scientific knowledge about the possible influence of the music in sport and exercise. We selected articles present in the database Physical Education Index, published between the years 2010 and 2015 and that were reviewed by experts. We excluded those that wouldn't treat the subject of this research and analyzed 13 articles. According to the studies reviewed, the music seems to acute benefit aerobic exercises, unlike the anaerobic exercises, which was probably by the high intensity. Nevertheless its effectiveness for the application in the sport, especially in high performance is not yet completely clear.

Keywords: Music. Exercise. Sport. Performance.

\section{La música en el deporte: revisión de la literatura}

\section{RESUMEN}

Independientemente de nuestros orígenes y contextos culturales, la música presenta papel fundamental en nuestras vidas. La música es capaz de cambios psicológicos y fisiológicos con aplicaciones en el deporte y ejercicio. El objetivo de este estudio es revisar el conocimiento científico sobre la posible influencia de la música en deporte y ejercicio. Artículos seleccionados en la base de datos Physical Education Index, publicado entre los años 2010 y 2015 y que fueron revisados por expertos. Fueran eliminados los que no trataban al tema de la presente investigación, así hemos analizado 13 artículos. Según los estudios revisados, la música parece beneficiar de forma aguda los ejercicios físicos de carácter aeróbico, a diferentemente de los ejercicios anaeróbicos, lo que se debe probablemente la alta intensidad. Sin embargo, su eficacia para el uso en el deporte, especialmente en el alto rendimiento aún no está totalmente claro.

Palabras Clave: Música. Deportes. Ejercicio. Rendimiento. 


\section{INTRODUÇÃO}

Independente de nossas origens e contextos culturais, a música apresenta papel fundamental em nossas vidas. Dessa forma, pesquisas científicas vêm documentando sua significativa influência no bem estar físico, psicológico e espiritual dos seres humanos (MINDLIN, DUROUSSEAU; CARDILLO, 20014).

Karageorghis e Terry (KARAGEORGHIS; TERRY, 2009) afirmaram que as pessoas têm tendência natural para responder ao ritmo e às qualidades motivacionais da música. Isso ocorre devido à ativação de diversas áreas cerebrais, como lobo temporal, córtex pré-frontal, cerebelo, hipocampo, amígdala, núcleo acumbens (MUSZAK, 2012; JACOB; FRANCONE; LOSSOW 1990), de modo a interferir nas percepções, emoções, memória, neuroquímica, comportamento e atividade do sistema nervoso autônomo ${ }^{1}$. Assim, a exposição à música provoca alterações fisiológicas, como frequência cardíaca, frequência respiratória, ritmos elétricos cerebrais, ciclo circadiano de sono-vigília, e psicológicas, como atenção, concentração, sensação de prazer, modulação da dor, sensação de calma e euforia, relaxamento e pensamentos (MINDLIN, DUROUSSEAU; CARDILLO, 2014; MUSZAK, 2012; CHATTERJEE, 2014; BERNARDI, 2006).

Considerando seus efeitos psicofisiológicos, a música apresenta aplicações no esporte e exercício, visando a melhora esportiva (KARAGEORGHIS; TERRY, 2009; TERRY; KARAGEORGHIS; 2006). Isso porque a música é capaz de captar a atenção, melhorar o ânimo, regular o humor, aumentar a produção de trabalho, reduzir as inibições e incentivar o movimento rítmico. É capaz ainda de reduzir a percepção subjetiva de esforço e os sinais de fadiga (KARAGEORGHIS; TERRY, 2009; CHATTERJEE, 2014; TERRY; KARAGEORGHIS; 2006; CARNEIRO et al., 2010; SOUZA; SILVA, 2010; YAMASHITA et al., 2006; MIRANDA; GODELI, 2003). Assim, é possível usar a música para atingir o rendimento esportivo ideal, através do alcance do estado de fluxo, estado ideal de atenção e concentração (MINDLIN, DUROUSSEAU; CARDILLO, 2014). Considerando o papel fundamental da música em nossas vidas, seus efeitos sobre o corpo e a mente e sua utilização no esporte, torna-se importante conhecer as produções científicas a respeito da música aplicada ao esporte e exercício. Neste contexto, o objetivo do presente estudo é revisar o conhecimento científico a respeito da possível influência da música no esporte e exercício.

\section{Metodologia}

Coletamos artigos na base de dados "Physical Education Index", a partir da busca pelas palavras chave "music and exercise", "music and sports" e "music and performance". A partir dos achados, como critério de inclusão, selecionamos os artigos publicados entre os anos de 2010 e 2015 e que foram revisados por especialistas 
(processo editorial que envolve revisão e aprovação de revisores críticos, na mesma área de atuação). Como critério de exclusão, não utilizamos artigos dos quais o título ou o resumo não tratavam do tema da nossa pesquisa.

\section{Resultados}

Encontramos 14 artigos e um foi excluído por não atender aos critérios definidos. Os dados dos 13 artigos selecionados foram extraídos e dispostos no Quadro 1.

Quadro 1 - Dados sobre a influência da música no esporte e exercício encontrados nos 13 artigos

\begin{tabular}{|c|c|c|c|c|c|}
\hline Autor (es) & Ano & Revista & Amostra & Modalidade & Resultados \\
\hline Silva et al. & 2015 & $\begin{array}{l}\text { Physiology and } \\
\text { Behavior }\end{array}$ & 14 homens & $\begin{array}{l}\text { Teste em ciclo } \\
\text { ergômetro }\end{array}$ & $\begin{array}{l}\text { Redução de sentimentos negativos. } \\
\text { Não houve aumento do rendimento } \\
\text { nem redução da PSE. }\end{array}$ \\
\hline $\begin{array}{l}\text { Bigliassi et } \\
\text { al. }\end{array}$ & 2015 & $\begin{array}{l}\text { Journal of Strength and } \\
\text { Conditioning Research }\end{array}$ & $\begin{array}{l}15 \text { corredores } \\
\text { amadores }\end{array}$ & Corrida & $\begin{array}{l}\text { Ativação da área pré-frontal. Melhora } \\
\text { dos estados de excitação e calma, PSE } \\
\text { e da recuperação. Não houve } \\
\text { diferença na FC. Há probabilidade de } \\
\text { efeito ergogênico. }\end{array}$ \\
\hline Yeats et al. & 2014 & Sport Science Review & 12 mulheres & Voleibol & $\begin{array}{l}\text { Melhora da percepção de motivação, } \\
\text { humor, coesão e desempenho. }\end{array}$ \\
\hline Atan & 2013 & Biology of Sport & 28 homens & $\begin{array}{l}\text { Testes em } \\
\text { esteira e ciclo } \\
\text { ergômetro }\end{array}$ & $\begin{array}{l}\text { Não houve diferença na potência } \\
\text { anaeróbia, FC e lactato sanguíneo. }\end{array}$ \\
\hline Terry et al. & 2012 & $\begin{array}{l}\text { Journal of Science and } \\
\text { Medicine in Sport }\end{array}$ & $\begin{array}{l}11 \text { ( } 6 \text { homens } \\
\text { e } 5 \text { mulheres) }\end{array}$ & Teste em esteira & $\begin{array}{l}\text { Melhora no tempo de exaustão e na } \\
\text { escala de sentimentos. Aumento da } \\
\text { PSE. A concentração de lactato } \\
\text { sanguíneo e o consumo de oxigênio } \\
\text { variaram durante os momentos da } \\
\text { coleta. }\end{array}$ \\
\hline Souza; Silva & 2012 & $\begin{array}{l}\text { Brazilian Journal of } \\
\text { Kinanthropometry and } \\
\text { Human Performance }\end{array}$ & 31 homens & Teste em esteira & $\begin{array}{l}\text { Não houve efeito crônico sobre o } \\
\text { rendimento. }\end{array}$ \\
\hline $\begin{array}{l}\text { Karageorghis } \\
\text { et al. }\end{array}$ & 2011 & $\begin{array}{l}\text { Research Quarterly for } \\
\text { Exercise and Sport }\end{array}$ & $\begin{array}{l}28 \text { ( } 15 \text { homens } \\
\text { e } 13 \text { mulheres })\end{array}$ & $\begin{array}{l}\text { Teste em ciclo } \\
\text { ergômetro }\end{array}$ & $\begin{array}{l}\text { Alteração da FC durante exercício } \\
\text { com condições musicais diferentes. }\end{array}$ \\
\hline $\begin{array}{l}\text { Laukka e } \\
\text { Quick }\end{array}$ & 2011 & Psychology of Music & $\begin{array}{l}252(117 \\
\text { homens e } 135 \\
\text { mulheres })\end{array}$ & $\begin{array}{l}\text { Modalidades } \\
\text { diversas }\end{array}$ & $\begin{array}{l}\text { Segundo respostas dos questionários, } \\
\text { a música melhora o desempenho, } \\
\text { motivação e estado de fluxo. }\end{array}$ \\
\hline Lane et al. & 2011 & $\begin{array}{l}\text { Journal of Sports } \\
\text { Science and Medicine }\end{array}$ & $\begin{array}{l}65 \text { ( } 19 \text { homens } \\
\text { e } 41 \text { mulheres) }\end{array}$ & Corrida & $\begin{array}{l}\text { Melhora nos estados emocionais e na } \\
\text { percepção de rendimento. }\end{array}$ \\
\hline $\begin{array}{l}\text { Brooks; } \\
\text { Brooks }\end{array}$ & 2010 & $\begin{array}{l}\text { Journal of Exercise } \\
\text { Physiology }\end{array}$ & $\begin{array}{l}71 \text { ( } 43 \text { homens } \\
\text { e } 28 \text { mulheres) }\end{array}$ & $\begin{array}{l}\text { Teste em ciclo } \\
\text { ergômetro }\end{array}$ & $\begin{array}{l}\text { Aumento da potência média e pico de } \\
\text { potência. }\end{array}$ \\
\hline $\begin{array}{l}\text { Waterhouse } \\
\text { et al. }\end{array}$ & 2010 & $\begin{array}{l}\text { Scandinavian Journal } \\
\text { of Medicine and } \\
\text { Science in Sports }\end{array}$ & 20 homens & $\begin{array}{l}\text { Teste em ciclo } \\
\text { ergômetro }\end{array}$ & $\begin{array}{l}\text { Música rápida aumentou o trabalho, a } \\
\text { FC e o tempo de duração do } \\
\text { exercício. }\end{array}$ \\
\hline Ghaderi et al & 2010 & $\begin{array}{l}\text { British Journal of } \\
\text { Sports Medicine }\end{array}$ & 30 homens & Teste em esteira & $\begin{array}{l}\text { Não houve alteração no desempenho } \\
\text { nem no cortisol salivar. }\end{array}$ \\
\hline $\begin{array}{l}\text { Karageorghis } \\
\text { et al. }\end{array}$ & 2010 & $\begin{array}{l}\text { Psychology of Sport } \\
\text { and Exercise }\end{array}$ & $\begin{array}{l}26 \text { ( } 13 \text { homens } \\
\text { e } 13 \text { mulheres })\end{array}$ & $\begin{array}{l}\text { Teste em } \\
\text { circuito }\end{array}$ & $\begin{array}{l}\text { Não houve alteração na potencia } \\
\text { anaeróbia; Houve respostas distintas } \\
\text { entre os gêneros. }\end{array}$ \\
\hline
\end{tabular}

Legenda: PSE - Percepção subjetiva de esforço; FC - Frequência cardíaca. 
Nosso estudo, por meio da revisão de literatura, analisou o conhecimento científico a respeito da influência da música no esporte e exercício. Como observado, nos últimos anos, trabalhos importantes foram produzidos a fim de sanar as incertezas sobre assunto. Apesar de classificada como recurso ergogênico por alguns autores (KARAGEORGHIS; TERRY, 2009; CARNEIRO et al., 2010), sua capacidade de melhorar o rendimento esportivo ainda não está clara, como mostram os resultados do presente estudo.

Os estudos analisados desenvolveram suas metodologias de acordo com diversas variáveis, como o teste aplicado (ciclo ergômetro, esteira, ambiente externo), as condições musicais utilizadas (música da preferência do participante, música escolhida pelos pesquisadores, músicas assíncronas, sincrônicas, rápida e lenta), o tempo de intervenção (apenas um estudo buscou efeito crônico), as variáveis psicológicas e fisiológicas (frequência cardíaca, percepção subjetiva de esforço, sentimentos, percepção de rendimento, concentração de cortisol salivar e lactato sanguíneo, potência, tempo de exaustão), as características da amostra (gênero, idade e nível de adaptação à modalidade) e principalmente, o tipo de exercício (exercícios aeróbios e anaeróbios),

\section{Exercícios aeróbios}

Em relação ao tipo de estudo, diversos autores (SILVA et al., 2015; BIGLIASSI et al.; TERRY et al. 2012; SOUZA; SILVA, 2012; LANE; DAVIS; DEVONPOR, 2011; WATERHOUSE; HUDSON; EDWARDS, 2010) analisaram a relação entre música e exercícios de caráter aeróbio.

Silva et al. (2015) examinaram o efeito da música (definida pelos autores) no tempo de exaustão e nas respostas psicofisiológicas em exercícios moderados, com e sem pré fadiga. Bigliassi et al. (2015). investigaram os efeitos da música (escolhida pelos participantes) no tempo de execução, ativação cerebral e respostas psicofisiológicas em um teste de $5 \mathrm{Km}$ de corrida. Terry et al. (2012) analisaram a música sincrônica sobre o desempenho de corrida em esteira de triatletas de elite. Souza e Silva (2012) verificaram a continuidade (período de quatro semanas) do efeito ergogênico da música assíncrona sobre o desempenho de corrida. Lane, Davis e Devonpor (2011) a partir de coleta online, compararam o efeito de duas intervenções diferentes de música (música síncrona e músicas motivacionais escolhidas pelos voluntários) sobre alterações emocionais e desempenho. Waterhouse, Hudson e Edwards (2010) avaliaram o rendimento dos voluntários em três condições musicais (ouvindo músicas com andamento padrão, ouvindo as mesmas músicas aceleradas 10\% e lentificada $10 \%$ ).

Com exceção do estudo de Souza e Silva (2012) que analisou o efeito crônico da música, os demais estudos demonstraram, de maneira geral, efeitos positivos da música em testes aeróbios. Esses achados corroboram com Karageorghis citado por Chatterjee, 
Gosh e Gosh (2014) que afirma que a música apresenta maior eficiência em exercícios de intensidade baixa e moderada.

Quanto à condição musical definida, com exceção de Silva et al. (2015), tanto as músicas escolhidas pelos autores quanto as da preferência dos voluntários, tiveram efeitos positivos sobre o rendimento e os estados emocionais, conforme sugerido por pesquisadores ingleses (CHATTERJEE; GOSH; GOSH, 2014).

Waterhouse, Hudson e Edwards (2010) foram os únicos a investigar a influência da música de diferentes andamentos. Seus resultados positivos para a seleção de música rápida em comparação com a seleção de música lenta corroboram com Mindlin, Durousseau e Cardillo (2014), que afirmaram que músicas aceleradas são estimulantes, ativando o cérebro e sincronizando os movimentos, provocando fluidez mental e muscular.

Como exposto, apesar de a literatura nos mostrar que a exposição à música é capaz de alterar padrões de variabilidade neurovegetativa (JACOB; FRANCONE; LOSSOW, 1990), reduzir a fadiga e a percepção subjetiva de esforço (MIRANDA; GODELI, 2003), os resultados para percepção subjetiva de esforço e frequência cardíaca ainda são controversos.

\section{Exercícios anaeróbios}

Buscando desvendar os efeitos da música em exercícios de caráter anaeróbio, Atan (2013), Brooks e Brooks (2010), Karageorghis et al. (2011) e Ghaderi et al. (2010) conduziram seus estudos.

Atan (2013) avaliou os efeitos da audição musical e seu ritmo em três condições (música rápida, lenta e sem música) sobre exercícios anaeróbios. Brooks e Brooks (2010) buscaram determinar se a música (escolhida pelos pesquisadores) melhora o rendimento no teste de Wingate. Karageorghis et al. (2010) analisaram os efeitos psicológicos e ergogênicos da música sincrônica motivacional em exercício de circuito. Ghaderi et al. (2010) determinaram os efeitos da música rápida e lenta no desempenho anaeróbio e na concentração de cortisol salivar. Karageorghis et al. (2011) investigaram a relação entre a frequência cardíaca no exercício (com variação de intensidade) com audição musical de tempo rápido.

De maneira geral, a música não se mostrou positiva sobre o rendimento quando aplicada em exercícios anaeróbios. Apenas Brooks e Brooks (2010) observaram melhora na potência em teste em ciclo-ergômetro.

Uma estratégia para tornar a música aliada para melhora do rendimento é ouvir músicas consideradas prazerosas, envolvendo variáveis como letra, melodia, 
associações, preferências, fundo sociocultural, entre outras, que interagem para determinar sua capacidade motivacional e sua eficiência provocar tais alterações (TERRY; KARAGEORGHIS; 2006; MIRANDA; GODELI, 2003; KARAGEORGHIS; TERRY; LANE, 1999, PRIEST; KARAGEORGHIS; SHARP, 2004). Das condições musicais utilizadas nos estudos, apenas Karagheorhis et al. (2010) selecionaram músicas condizentes às condições motivacionais para sua amostra, mas não foi suficiente para melhorar o desempenho. A possível explicação é de que durante exercícios de alta intensidade, os sinais de estresse se intensificam, chamando atenção para os sinais fisiológicos de fadiga (CHATTERJEE; GHOSH; GHOSH, 2014) tornando os efeitos da música pouco eficazes.

Em relação às reações psicológicas, apenas Karageorghis et al. (2010) avaliaram respostas afetivas após o teste, e não observaram diferença entre as condições musicais. No entanto, encontraram diferença nas respostas entre os gêneros da amostra, corroborando com Priest, Karageorghis e Sharp. Para as reações fisiológicas, apenas Karageorghis et al. (2011) observaram alteração na frequência cardíaca com música rápida em algumas intensidades de exercício, em comparação com o teste sem audição musical. Enquanto nos demais estudos não houve alteração na frequência cardíaca, concentração de lactato sanguíneo e cortisol salivar.

Dentre outras modalidades esportivas e de coleta de dados subjetivos, Laukka e Quick (2011), por meio de aplicação de questionário eletrônico, obtiveram dados sobre os diferentes usos da música, com foco motivacional e emocional no esporte e exercício. Yeats et al. (2014) analisaram a percepção de jogadores de voleibol do ensino médio e seus técnicos sobre os efeitos de uma seleção musical no aquecimento antes da competição (warm-up). Em ambos os estudos, houve percepção de melhora de rendimento esportivo e motivação.

\section{CONSIDERAÇÕES FINAIS}

A música parece beneficiar de forma aguda os exercícios físicos de caráter aeróbio, enquanto não apresenta qualquer influência sobre exercícios anaeróbios. Apesar disso, sua eficácia para a aplicação no treinamento físico e na busca por melhora no rendimento esportivo ainda não está completamente clara. Isso se deve às inúmeras variáveis envolvidas em tais efeitos, o que amplia o leque de aplicações em pesquisas. Vale ressaltar ainda que as variáveis pessoais podem influenciar diferentemente as pessoas; assim, os resultados de um estudo podem não ser aplicáveis a um grande contingente populacional. Por isso, há ainda grande importância no incentivo a mais estudos na área, buscando cercar mais variáveis a fim de encontrar resultados consistentes no que tange às condições musicais (músicas autos selecionadas ou com a velocidade determinada pela percepção dos voluntários), nível dos praticantes, tipo de 
exercícios (aeróbios, anaeróbios e de força) e modalidades (coletivas e individuais), que sejam aplicáveis na pratica esportiva. Além disso, pesquisas que avaliem as respostas agudas e crônicas do treinamento com música, aliando instrumentos de quantificação de motivação, de percepção de rendimento e de atividade cerebral, podem trazer grandes descobertas e assim, melhorar a lacuna científica no tema.

\section{REFERÊNCIAS}

ATAN, Tugba. Effect of music on anaerobic exercise performance. Biology of Sport, v. 30, n. 1, p. 35-39, 2013.

BERNARDI, Luciano; PORTA, Camillo; SLEIGHT, Peter. Cardiovascular, cerebrovascular, and respiratory changes induced by different types of music in musicians and non-musicians: the importance of silence. Heart, London, v. 92, n. 4, p. 445-452, 2006.

BIGLIASSI, Marcelo et al. How does music aid $5 \mathrm{~km}$ of running? Journal of Strength Conditioning Research, Colorado Springs, v. 29, n. 2, p. 305-314, 2015.

BROOKS, Kelly; BROOKS, Kristal. Difference in wingate power output in response to music as motivation. Journal of Exercise Physiology Online, v. 13, n. p. 14-20, 2010. Disponível em: $<$ https://www.asep.org/asep/asep/JEPonlineDec2010Brooks.docx +\&cd=1\&hl=ptBR\&ct=clnk\&gl=br.>. Acesso em: 26 fev. 2016.

CARNEIRO, João Guilherme et al. Música: recurso ergogênico psicológico durante o exercício físico?. Revista Brasileira de Psicologia do Esporte, Brasília, v. 3, n. 2, p. 61$70,2010$.

CHATTERJEE, Saikot; GHOSH, Madhab Chandra; GHOSH, Arnab. A study to explore the effects of varied tempo music on brisk walking performance. Journal of Sports and Physical Education, v. 1, n. 7, p. 43-46, nov./dec. 2014.

GHADERI, Mohammad et al. Influence of music type listening on anaerobic performance and salivary cortisol in males athletes. British Journal of Sports Medicine, Loughborough, v. 44, n. 1, p. 162-167, 2010. Disponível em: <https://www.researchgate.net/publication/254744599_The_effect_of_fast_and_slow_r hythm_music_on_anaerobic_performance_and_salivary_cortisol_in_athlete_males.>. Acesso em: 26 fev. 2016. 
JACOB, Stanley W.; FRANCONE, Clarice Ashworth; LOSSOW, Walter J. Anatomia e fisiologia humana. 5. ed. Rio de Janeiro: Guanabara,1990. p. 278-299.

KARAGEORGHIS, Costas I.; TERRY, Peter C. The psychological, psychophysical and ergogenic effects of music in sport: a review and synthesis. In: BATEMAN, A. J.; BALE, J. R. (Ed.). Sporting sounds: relationships between sport and music. London: Routledge, 2009. p. 13-36. Disponível em:

<http://bura.brunel.ac.uk/bitstream/2438/6700/2/FullText.pdf>. Acesso em: fev. 2016.

KARAGEORGHIS, Costas I. TERRY, Peter C.; LANE, Andrew. Development and initial validation of an instrument to assess the motivational qualities of music in exercise and sport: the brunel music rating inventory. Journal of Sports Sciences, London, v. 17, n. 9, p. 713-724, 1999.

KARAGEORGHIS, Costas I. et al. Ergogenic and psychological effects of synchronous music during circuit-type exercise. Psychology of Sport and Exercise, Netherlands, v. 11, n. 6, p. 551-559, 2010.

KARAGEORGHIS, Costas I. et al. Revisiting the relationship between exercise heart rate and music tempo preference. Research Quarterly for Exercise and Sport, Reston, v. 82, n. 2, p. 274-284, 2011.

LANE, Andrew M.; DAVIS, Paul A.; DEVONPOR, Tracey J. Effects of music interventions on emotional states and running performance. Journal of Sports Science and Medicine, Bursa, v. 10, n. 2, p. 400-407, 2011.

LAUKKA, Petri; QUICK, Lina. Emotional and motivational uses of music in sports and exercise: A questionnaire study among athletes. Psychology of Music, London, v. 41, n. 2, p. 198-215, 2011.

MINDLIN, Galina; DUROUSSEAU, Don; CARDILLO, Joseph. Sua playlist pode mudar sua vida. Rio de Janeiro: BestSeller, 2014.

MIRANDA, Maria Luiza de Jesus; GODELI, Maria Regina C. Souza. Música, atividade e bem-estar psicológico em idosos. Revista Brasileira de Ciência e Movimento, Brasília, v. 11, n. 4, p. 87-94, 2003.

MUSZKAT, Mauro. Música, neurociência e desenvolvimento humano. In: BRASIL. Ministério da Cultura. A música na escola. Brasília, 2012. p. 67-71. Disponível em: <http://www.amusicanaescola.com.br/>. Acesso em: 26 fev. 2016.

PRIEST, David Lee; KARAGEORGHIS, Costas I.; SHARP, N. C. Craig. The characteristics and effects of motivational music in exercise settings: the possible 
influence of gender, age, frequency of attendance and time of attendance. Journal of Sports Medicine and Physical Fitness, Torino, v. 44, n. 1, p. 77-86, 2004.

SILVA, João P. Lopes et al. Influence of music on performance and psychophysiological responses during moderate-intensity exercise preceded by fatigue. Physiology and Behavior, Elmsford, v. 139, p. 274-280, 2015.

SOUZA, Yonel Ricardo; SILVA, Eduardo Ramos. Efeitos psicofísicos da música no exercício: uma revisão. Revista Brasileira de Psicologia do Esporte, Brasília, v. 3, n. 2, p. 33-45, 2010.

SOUZA, Yonel Ricardo; SILVA, Eduardo Ramos. Temporal analysis of the ergogenic effect of asynchronous music on exercise. Revista Brasileira de Cineantropometria e Desempenho Humano, Florianópolis, v. 14, n. 3, p. 305-312, 2012.

TERRY, Peter C.; KARAGEORGHIS, Costas I. Psychophysical effects of music in sport and exercise: an update on theory, research and application. In: KATSIKITIS, M. Psychology bridging the Tasman: science, culture and practice. Melbourne: Australian Psychological Society, 2006. Disponível em: <https://www.piuvivi.com/docs/effettimusica-sulla-psiche.pdf >. Acesso em: 26 fev. 2016.

TERRY, Peter C. et al. Effects of synchronous music on treadmill running among elite triathletes. Journal of Science and Medicine in Sport, Belconnen, v. 15, n. 1, p. 52-57, 2012.

WATERHOUSE, Jim; HUDSON, Pollyana; EDWARDS, Ben. Effects of music tempo upon submaximal cycling performance. Scandinavian Journal of Medicine and Science in Sports, v. 20, n. 4, p. 662-669, 2010.

YAMASHITA, Saori et al. Effects of music during exercise on RPE, heart rate and the autonomic nervous system. Journal of Sports Medicine and Physical Fitness, Turin, v. 43, n. 3, p. 425-430, 2006.

YEATS, Jeremy et al. High school volleyball athletes: perceptions of creating and using pre-competition warm-up music. Sport Science Review, Champaign, v. 23, n. 3/4, p. 127-150, 2014. 